\title{
Biased PNG Law for Impact-Time Control
}

\author{
By Tae-Hun KIM, ${ }^{1)}$ Chang-Hun LeE, ${ }^{1)}$ Min-Jea TAHK ${ }^{2)}$ and In-Soo JeON ${ }^{1)}$ \\ ${ }^{1)}$ Agency for Defense Development (ADD), Daejeon, Korea \\ ${ }^{2)}$ Department of Aerospace Engineering, Korea Advanced Institute of Science and Technology (KAIST), Daejeon, Korea
}

(Received May 2nd, 2012)

\begin{abstract}
This paper proposes a biased proportional navigation guidance (PNG) law to control the impact time, that can be used for salvo attacks or cooperative missions of missiles. To derive the guidance command of the proposed law, we introduce a polynomial function of the command with two coefficients, one of which is to achieve zero miss-distance and the other is to satisfy the terminal impact time constraint. Since the proposed guidance law has an arbitrary guidance gain, it is possible to shape the homing trajectory and acceleration command profile by choosing a proper guidance gain in consideration of the missile's capability and operational conditions. Numerical simulations for the various forms of the guidance command are performed to investigate the characteristics and performance of the proposed law.
\end{abstract}

Key Words: Impact-Time Control, Proportional Navigation Guidance, Biased PNG, Polynomial Function

\section{Introduction}

In order to protect one's own ship against anti-ship missiles, enemy aircraft and other warfare threats, modern battleships are equipped with a number of self-defense systems, such as anti-air defense missiles, an electronic countermeasures (ECM) system and an advanced close-in weapon system (CIWS). In particular, the CIWS can automatically detect, evaluate, track, engage and destroy at short range incoming anti-ship missiles that have penetrated the outer defenses. Since these defense systems provide powerful self-defense capabilities with a fast-reaction, anti-ship missile developers have devised several ways that can enhance the survivability of the anti-ship missiles against advanced defense systems. One of these ways is a salvo attack that involves multiple missiles hitting the same target as simultaneously as possible. Since the CIWS of the battleship might be vulnerable to a many-to-one engagement situation in which multiple missiles approach simultaneously to one CIWS, a salvo attack could reduce the probability of the missile being intercepted by the projectiles of CIWS guns, and consequently it could improve the survivability of the missiles. To the salvo attack, the missiles performing the cooperative attack mission should be able to hit the target at the designated impact time; that is, terminal impact time constraint should be considered for homing missile guidance laws. ${ }^{1,2)}$ For the formation flight of unmanned aerial vehicles (UAVs), the timing constraint, which is the synchronization of the arrival time at a rendezvous point, is also considered in order to increase mission effectiveness, minimize the formation delay, and so on. ${ }^{3-8)}$

Although studies on guidance problems with terminal impact time constraint for homing missiles have attracted more attention in recent years, only a few guidance laws to control the impact time have been developed so far. ${ }^{1,2,9-13)}$ The pro-

(C) 2013 The Japan Society for Aeronautical and Space Sciences posal in Ref. 1) for a suboptimal guidance law with an impact time constraint appears to be the first attempt to design an impact time control guidance law. The authors also proposed a new guidance law to control the impact time as well as the terminal impact angle using the jerk control term based on the optimal control theory. ${ }^{2)}$ In Ref. 9), the proportional navigation guidance (PNG) law with a timevarying navigation gain, called cooperative proportional navigation $(\mathrm{CPN})$, was suggested to make the missiles intercept the target at the same arrival time. In this guidance problem, the navigation gains for each missile are adjusted to synchronize the arrival times by communicating among the missiles without designated impact times. A homing guidance law, which is a combination of the well-known optimal impact angle control law and an additional command to meet the impact time constraint, was also proposed by the same authors mentioned above. ${ }^{10)}$ Based on nonlinear control design methods, guidance laws with terminal impact time and impact angle constraints were developed in Refs. 11)-13). In Ref. 11), the feedback linearization method was used to obtain an impact time control law, and the backstepping control method and modified PNG law were utilized to design the impact time and angle controller in Ref. 12). The impact time and angle control law in Ref. 13) was derived from the proposed line-of-sight (LOS) rate shaping technique and second-order sliding mode control, where both the LOS angle and rate profiles are determined to satisfy the terminal constraints and then the sliding mode control is designed to track the obtained LOS rate profile.

Note that the conventional PNG law has been widely used as a homing guidance method because of its ease of implementation and efficiency, and the navigation constant $N$ of the PNG law is chosen in the range from 3 to 5 . Although the PNG with $N=3$ is known as the energy optimal solution, ${ }^{14,17,18)}$ arbitrary $N>3$ is also recommended in practice 
to improve the guidance performance under disturbances and uncertainties such as initial position/heading errors, sensor noises, target's maneuvering capability, and so on. ${ }^{20,21)}$ For these reasons, therefore, the derivation of the impact time control law with an arbitrary guidance gain is meaningful in terms of the practical implementation, and it can provide systematic selection of guidance gains for missile system designers. The suboptimal impact-time control law of Ref. 1), which was derived on the basis of the optimal control theory and a linear formulation, consists of the conventional PNG law with navigation constant $N=$ 3 and the additional bias term to modulate the homing trajectory for satisfying the terminal impact time constraint; that is, the suboptimal guidance law can be represented as a biased PNG (BPNG) law with $N=3$. In this paper, we extend this suboptimal impact-time control law to a more generalized BPNG law, which can control arbitrary impact times and furthermore has arbitrary navigation constant other than $N=3$.

To derive the BPNG law to control the impact time with arbitrary gains, we suggest a simple approach, in which the guidance command is assumed to be represented as a polynomial function of the downrange-to-go and the coefficients of the assumed function are then determined to satisfy the terminal impact time constraint as well as zero terminal miss-distance. Using the determined coefficient solutions, we can finally obtain the state feedback guidance law which is expressed as a combination of the PNG law for reducing the miss-distance and the additional bias term for adjusting the length of the homing trajectory.

This paper is organized as follows: In section 2, the nonlinear homing problem and boundary conditions with terminal impact time constraint are defined. In sections 3 and 4, a biased PNG law with an unspecified guidance gain that can achieve the designated impact time is derived using the proposed simple approach. Section 5 presents numerical simulations conducted to evaluate the performance and characteristics of the proposed guidance law. Finally, section 6 provides the conclusions of this work.

\section{Problem Statement}

Consider the two-dimensional planar homing engagement geometry of a missile $M$ against a stationary target $T$, as depicted in Fig. 1. In this engagement, we assume that the missile velocity $V$ is constant, and the autopilot lag is negligible. Here, $(x, y)$ and $\theta$ represent the missile positions with respect to the inertial reference frame $(X-Y)$ and the flight path angle, respectively. The missile acceleration command normal to the velocity is denoted by $a$. From Fig. 1, the nonlinear equations of motion with respect to the time, $t$, for this homing problem are given as

$$
\begin{array}{lll}
\dot{x}=V \cos \theta, & x\left(t_{0}\right)=x_{0}, & x\left(t_{d}\right)=x_{f} \\
\dot{y}=V \sin \theta, & y\left(t_{0}\right)=y_{0}, & y\left(t_{d}\right)=y_{f} \\
\dot{\theta}=a / V, & \theta\left(t_{0}\right)=\theta_{0} &
\end{array}
$$

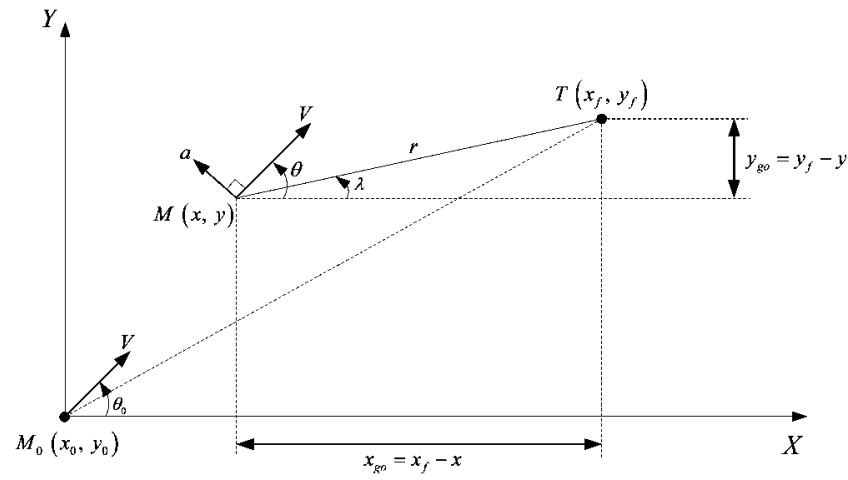

Fig. 1. Homing engagement geometry.

where $t_{0}$ is the initial time and $t_{d}$ is the desired impact time which is designated before the missile is launched.

If the flight path angle, $\theta$, and the initial line-of-sight angle, $\lambda_{0}$, are small, the linearized equations of motion with respect to $x$ can be obtained as

$$
\left[\begin{array}{l}
y^{\prime} \\
\theta^{\prime}
\end{array}\right]=\left[\begin{array}{ll}
0 & 1 \\
0 & 0
\end{array}\right]\left[\begin{array}{l}
y \\
\theta
\end{array}\right]+\left[\begin{array}{l}
0 \\
1
\end{array}\right] u
$$

where the prime' denotes the derivative with respect to $x$ and the guidance command is defined by $u \triangleq a / V^{2}$. The initial and final boundary conditions for these linearized equations are given as

$$
y\left(x_{0}\right)=y_{0}, \quad \theta\left(x_{0}\right)=\theta_{0}, \quad y\left(x_{f}\right)=y_{f} .
$$

Note that the final boundary condition, $y\left(x_{f}\right)=y_{f}$, is required to hit the target at $x=x_{f}$. In the nonlinear equations of motion given in Eq. (1), the terminal constraints, $x\left(t_{d}\right)=x_{f}, y\left(t_{d}\right)=y_{f}$, are defined for the successful interception of the target at the desired impact time. The missile positions at the desired impact time should be equal to the target positions. That is, the total homing flight time should be equal to the desired impact time. However, in the linearized equations of motion, this terminal impact time constraint can be replaced by a path constraint that the total length of the intercept trajectory should be the same as the desired distance-to-go, $\hat{r}_{\mathrm{go}}^{*}$, where $\hat{r}_{\mathrm{go}}^{*}=V\left(t_{d}-t_{0}\right)$, and this path constraint is valid because the missile velocity, $V$, is constant.

In addition to the final boundary condition given in Eq. (3), therefore, we consider the following path constraint for the impact-time control.

$$
S=\int_{x_{0}}^{x_{f}} \sqrt{1+\left(y^{\prime}\right)^{2}} \mathrm{~d} \eta \approx \int_{x_{0}}^{x_{f}} 1+\frac{1}{2} \theta^{2}(\eta) \mathrm{d} \eta=\hat{r}_{\mathrm{go}}^{*}
$$

where $S$ is the total length of the intercept trajectory from $x_{0}$ to $x_{f}$, and can be approximated as Eq. (4) because the flight path angle is assumed to be small in the linearized equations.

Let us consider the closed-form solution of the PNG law, which is a well-known homing guidance method presented in Ref. 20). The closed-form solution for the missile acceleration, which is derived using the small angle approximation, is expressed as 


$$
\left.a_{\mathrm{PNG}}(t)\right|_{\mathrm{sol}}=\frac{N\left[\hat{y}_{\mathrm{go}}+\dot{\hat{y}}_{\mathrm{go}} t_{f}\right]}{t_{f}^{N}}\left(t_{f}-t\right)^{N-2}
$$

where $N$ is the navigation constant and $t_{f}$ is the flight time. $\hat{y}_{\text {go }}=y_{f}-y_{0}$ and $\dot{\hat{y}}_{\text {go }}=-V \theta_{0}$ are the initial relative crossrange and initial relative lateral velocity perpendicular to the $X$-axis, respectively. Using $x_{f}=V t_{f}$ and $x=V t$, the solution of Eq. (5) can be rewritten in terms of $x$ for the given linearized equations of Eq. (2).

$$
\left.u_{\mathrm{PNG}}(x)\right|_{\mathrm{sol}}=\frac{\left.a_{\mathrm{PNG}}(t)\right|_{\mathrm{sol}}}{V^{2}}=\frac{N\left[\hat{y}_{\mathrm{go}}-\theta_{0} x_{f}\right]}{x_{f}^{N}}\left(x_{f}-x\right)^{N-2}
$$

As shown in Eq. (6), the PNG command history for reducing the miss-distance is represented by the $(N-2)$ th-order function of the downrange-to-go, $x_{\mathrm{go}}=x_{f}-x$.

In the homing problem with the terminal impact time constraint, there are two terminal constraints: zero missdistance of Eq. (3) and the terminal impact time constraint (i.e., the path constraint given by Eq. (4)). The conventional PNG law can achieve the zero miss-distance, but it cannot achieve the terminal impact time constraint; that is, the guidance command history given by Eq. (6) can only satisfy the terminal zero miss-distance when the navigation constant is fixed. Therefore, in order to satisfy the additional constraint on the terminal impact time, the guidance command history of Eq. (6) should be modified appropriately. As mentioned above, the PNG command history for hitting the target is expressed as the downrange-to-go to the $(N-2)$ th power. Based on this relationship, let the guidance command history for the impact-time control be defined as the following polynomial function with a constant bias $c_{m}$.

$$
\left.u_{\mathrm{BPNG}}(x)\right|_{\mathrm{sol}}=c_{n} x_{\mathrm{go}}^{n}+c_{m}, \quad n=N-2>0
$$

where $n$ is an arbitrary positive constant; that is, $N>2$. Note that, as shown in Eq. (7), the guidance command profile is assumed to be represented as the polynomial function with two unknown coefficients in order to obtain a unique solution of the guidance law and provide one more degree of freedom by the constant coefficient $c_{m}$. The coefficient $c_{n}$ in the first term of the assumed function is considered for intercepting the target (i.e., zero miss-distance) and the second constant coefficient $c_{m}$ is used for achieving the desired impact time (i.e., the path constraint).

Using the assumed guidance command history in Eq. (7), we derive a state feedback guidance law that can satisfy the terminal constraints on the miss-distance and impact time in following sections.

\section{Guidance Command for Target Interception}

\subsection{Feedback command for target interception}

To obtain the state feedback guidance law for successful interception of the target, we should first determine the coefficient $c_{n}$ in the assumed guidance command history. Let us substitute Eq. (7) into Eq. (2) and then integrate the resulting equation with the initial conditions. After that, we can obtain

$$
y(x)=\frac{1}{2} c_{m} x^{2}+\frac{1}{(n+1)(n+2)} c_{n} x_{\mathrm{go}}^{n+2}+c_{\theta} x+c_{y}
$$

where $c_{\theta}$ and $c_{y}$ are the constants of integration, which are defined as

$$
\begin{aligned}
c_{\theta}= & \theta_{0}-c_{m} x_{0}+\frac{1}{(n+1)} c_{n}\left(x_{f}-x_{0}\right)^{n+1} \\
c_{y}= & y_{0}-\frac{1}{2} c_{m} x_{0}^{2}-\frac{1}{(n+1)(n+2)} c_{n}\left(x_{f}-x_{0}\right)^{n+2} \\
& -c_{\theta} x_{0}
\end{aligned}
$$

From Eqs. (8) and (9), the missile $y$ position at $x=x_{f}$ is

$$
\begin{aligned}
y\left(x_{f}\right) & =\frac{1}{2} c_{m} x_{f}^{2}+c_{\theta} x_{f}+c_{y} \\
& =\frac{1}{2} c_{m} \hat{x}_{\mathrm{go}}^{2}+\theta_{0} \hat{x}_{\mathrm{go}}+\frac{1}{(n+2)} c_{n} \hat{x}_{\mathrm{go}}^{n+2}+y_{0}
\end{aligned}
$$

where $\hat{x}_{\mathrm{go}}=x_{f}-x_{0}$. For the terminal zero miss-distance, the final missile position $y\left(x_{f}\right)$ should satisfy the final boundary condition, $y\left(x_{f}\right)=y_{f}$, given in Eq. (3). Using Eq. (10) and this boundary condition, we can determine the coefficient $c_{n}$ at $x=x_{0}$ to intercept the target as follows.

$$
c_{n}=\frac{(n+2)}{\hat{x}_{\mathrm{go}}^{n+2}}\left(\hat{y}_{\mathrm{go}}-\theta_{0} \hat{x}_{\mathrm{go}}-\frac{1}{2} c_{m} \hat{x}_{\mathrm{go}}^{2}\right)
$$

Hence, substituting Eq. (11) into Eq. (7) yields the guidance command at the initial $x_{0}$ as

$$
u_{\mathrm{BPNG}}\left(x_{0}\right)=(n+2) \frac{\left(\hat{y}_{\mathrm{go}}-\theta_{0} \hat{x}_{\mathrm{go}}\right)}{\hat{x}_{\mathrm{go}}^{2}}-\frac{n}{2} c_{m}
$$

If the coefficient $c_{n}$ is initialized and recalculated at each step of $x$, the guidance command given in Eq. (12) can be expressed in the form of the state feedback as

$$
u_{\mathrm{BPNG}}(x)=u_{p}(x)-\frac{n}{2} c_{m}
$$

where

$$
u_{p}(x)=(n+2) \frac{\left(y_{\mathrm{go}}-\theta x_{\mathrm{go}}\right)}{x_{\mathrm{go}}^{2}}
$$

and $y_{\text {go }}=y_{f}-y$. The feedback guidance command given in Eq. (13) enables the missile to hit the target even though the coefficient $c_{m}$ has any arbitrary constant. In next subsection, we derive the closed-form solutions of $u_{\mathrm{BPNG}}$ of Eq. (13) to investigate the characteristics of the obtained feedback guidance law. These closed-form solutions can also be used for calculating the total homing trajectory length of the missile driven by $u_{\mathrm{BPNG}}$.

\subsection{Closed-form solutions}

The closed-form solutions of $u_{\mathrm{BPNG}}$ can be obtained by solving the following linear second-order ordinary differential equation (ODE), which is determined by substituting Eq. (13) into Eq. (2).

$$
y^{\prime \prime}+\frac{(n+2)}{\left(x_{f}-x\right)} y^{\prime}-\frac{(n+2)}{\left(x_{f}-x\right)^{2}}\left(y_{f}-y\right)=-\frac{n}{2} c_{m}
$$


For convenience, the above second-order ODE is rewritten using $Y=y_{f}-y$ and $\tau=x_{f}-x$.

$$
D^{2} Y-\frac{(n+2)}{\tau} D Y+\frac{(n+2)}{\tau^{2}} Y=\frac{n}{2} c_{m}
$$

where $D$ is a differentiation operator with respect to the independent variable $\tau$. This equation is the same form as the Euler-Cauchy equation, ${ }^{19)}$ so we can easily find the solutions of Eq. (16).

The homogeneous solution to the ODE is

$$
Y_{h}(\tau)=c_{1} \tau^{n+2}-c_{2} \frac{1}{(n+1)} \tau
$$

and the particular solution to the ODE is

$$
Y_{p}(\tau)=-\frac{1}{2} c_{m} \tau^{2}
$$

Hence, from Eqs. (17) and (18), the general solutions of Eq. (15) can be obtained as

$$
\begin{aligned}
\left.y(x)\right|_{\mathrm{sol}} & =y_{f}-c_{1} x_{\mathrm{go}}^{n+2}+c_{2} \frac{1}{(n+1)} x_{\mathrm{go}}+\frac{1}{2} c_{m} x_{\mathrm{go}}^{2} \\
\left.\theta(x)\right|_{\mathrm{sol}} & =\left.y^{\prime}(x)\right|_{\mathrm{sol}} \\
& =c_{1}(n+2) x_{\mathrm{go}}^{n+1}-c_{2} \frac{1}{(n+1)}-c_{m} x_{\mathrm{go}} \\
\left.u(x)\right|_{\mathrm{sol}} & =\left.\theta^{\prime}(x)\right|_{\mathrm{sol}} \\
& =-c_{1}(n+2)(n+1) x_{\mathrm{go}}^{n}+c_{m} \\
& =c_{n} x_{\mathrm{go}}^{n}+c_{m}
\end{aligned}
$$

where $c_{1}$ and $c_{2}$ are the constants of integration

$$
\begin{aligned}
& c_{1}=\frac{-1}{(n+1)(n+2) \hat{x}_{\mathrm{go}}^{n}} u_{p}\left(x_{0}\right)+\frac{1}{2(n+1) \hat{x}_{\mathrm{go}}^{n}} c_{m} \\
& c_{2}=-\hat{x}_{\mathrm{go}} u_{p}\left(x_{0}\right)-(n+1) \theta_{0}-\frac{n}{2} c_{m} \hat{x}_{\mathrm{go}}
\end{aligned}
$$

From Eq. (19), it can be seen that the final boundary condition given by Eq. (3) can be always satisfied for an arbitrary $c_{m}$, and therefore, the feedback command $u_{\mathrm{BPNG}}$ can achieve the terminal zero miss-distance. It is also seen that the closed-form solution $\left.u(x)\right|_{\text {sol }}$ of the feedback command is identical to the assumed guidance command history given by Eq. (7), as expected. As shown in $\left.\theta(x)\right|_{\text {sol }}$, the missile flight path angle is dependent on the constant coefficient $c_{m}$. This result implies that the flight path angle can be adjusted by choosing the coefficient $c_{m}$. Using the coefficient $c_{m}$, therefore, we can shape the homing trajectory without violation of the terminal zero miss-distance. Accordingly, in the next section, we determine the appropriate coefficient $c_{m}$ to satisfy the path constraint for the impact-time control.

\section{Guidance Command for Impact-Time Control}

\section{1. $c_{m}$ for impact-time control}

To determine the coefficient $c_{m}$ that can meet the path constraint, we first derive the total length of the intercept trajectory generated by $u_{\mathrm{BPNG}}$. From Eq. (4), the total length of the intercept trajectory estimated from the initial $x_{0}$ to the final downrange $x_{f}$ can be rewritten as

$$
S=\hat{x}_{\mathrm{go}}+\frac{1}{2} \int_{x_{0}}^{x_{f}} \theta^{2}(\eta) \mathrm{d} \eta
$$

By substituting the solution $\theta$ of Eq. (19) into Eq. (21) and manipulating the resulting equations, the integral term of the right-hand side of Eq. (21) can be obtained as

$$
\begin{aligned}
\frac{1}{2} \int_{x_{0}}^{x_{f}} \theta^{2}(\eta) \mathrm{d} \eta= & \frac{n^{2}}{12(2 n+3)(n+3)} \hat{x}_{\mathrm{go}}^{3} c_{m}^{2} \\
& +\frac{n}{2(n+2)(n+3)(2 n+3)} \\
& \times \hat{x}_{\mathrm{go}}^{3} u_{p}\left(x_{0}\right) c_{m} \\
& +\frac{1}{(2 n+3)(n+2)} \hat{x}_{\mathrm{go}}^{3} u_{p}^{2}\left(x_{0}\right) \\
& +\frac{1}{(n+2)} \hat{x}_{\mathrm{go}}^{2} \theta_{0} u_{p}\left(x_{0}\right)+\frac{1}{2} \theta_{0}^{2} \hat{x}_{\mathrm{go}}
\end{aligned}
$$

Hence, from Eqs. (21) and (22), we have

$$
S=p_{1} \hat{x}_{\mathrm{go}}^{3} c_{m}^{2}+p_{2} \hat{x}_{\mathrm{go}}^{3} u_{p}\left(x_{0}\right) c_{m}+\left.\hat{r}_{\mathrm{go}}\right|_{c_{m}=0}
$$

where

$$
\begin{aligned}
p_{1}= & n^{2} /[12(2 n+3)(n+3)] \\
p_{2}= & n /[2(n+2)(n+3)(2 n+3)] \\
\left.\hat{r}_{\mathrm{go}}\right|_{c_{m}=0}= & \frac{1}{(2 n+3)(n+2)} \hat{x}_{\mathrm{go}}^{3} u_{p}^{2}\left(x_{0}\right) \\
& +\frac{1}{(n+2)} \hat{x}_{\mathrm{go}}^{2} \theta_{0} u_{p}\left(x_{0}\right)+\left(1+\frac{1}{2} \theta_{0}^{2}\right) \hat{x}_{\mathrm{go}}
\end{aligned}
$$

In Eq. (24), $\left.\hat{r}_{\text {go }}\right|_{c_{m}=0}$ is the length of the intercept trajectory of the missile guided by $u_{\mathrm{BPNG}}$ with $c_{m}=0$; that is, $\left.\hat{r}_{\mathrm{go}}\right|_{c_{m}=0}$ is the estimated distance-to-go using $u_{p}(x)$ at the initial $x_{0}$.

As mentioned in the previous section, $S$ should be equal to the desired distance-to-go, $\hat{r}_{\mathrm{go}}^{*}$, in order to make the missile hit the target at the designated impact time. Therefore, from Eqs. (4) and (23), we can obtain the following equation for determining the coefficient $c_{m}$.

$$
p_{1} \hat{x}_{\mathrm{go}}^{3} c_{m}^{2}+p_{2} \hat{x}_{\mathrm{go}}^{3} u_{p}\left(x_{0}\right) c_{m}-\hat{\varepsilon}=0
$$

where the distance-to-go error $\hat{\varepsilon}=\hat{r}_{\mathrm{go}}^{*}-\left.\hat{r}_{\mathrm{go}}\right|_{c_{m}=0}$, and this equation is a quadratic equation in terms of $c_{m}$. Hence, the solutions of Eq. (25) are given by

$$
\begin{aligned}
c_{m} & =\frac{1}{2}\left[-\frac{p_{2}}{p_{1}} u_{p}\left(x_{0}\right) \pm \sqrt{\left(\frac{p_{2}}{p_{1}}\right)^{2} u_{p}^{2}\left(x_{0}\right)+\frac{4}{p_{1} \hat{x}_{\mathrm{go}}^{3}} \hat{\varepsilon}}\right] \\
& =\frac{1}{2}\left[-\frac{p_{2}}{p_{1}} u_{p}\left(x_{0}\right) \pm\left|u_{p}\left(x_{0}\right)\right| \sqrt{\left(\frac{p_{2}}{p_{1}}\right)^{2}+\frac{4}{p_{1} u_{p}^{2}\left(x_{0}\right) \hat{x}_{\mathrm{go}}^{3}}} \hat{\varepsilon}\right]
\end{aligned}
$$

The coefficient $c_{m}$ has two solutions, as shown in Eq. (26). If $\left.\hat{r}_{\mathrm{go}}\right|_{c_{m}=0}=\hat{r}_{\mathrm{go}}^{*}$, then $c_{m}$ is not required for the impact-time control; that is, $c_{m}=0$ when $\hat{\varepsilon}=0$. From this condition, we can choose the unique $c_{m}$ as follows.

$$
c_{m}=-\frac{p_{2}}{2 p_{1}} u_{p}\left(x_{0}\right)\left[1-\sqrt{1+\frac{4 p_{1}}{p_{2}^{2} u_{p}^{2}\left(x_{0}\right) \hat{x}_{\mathrm{go}}^{3}} \hat{\varepsilon}}\right]
$$

The solution of $c_{m}$ obtained in Eq. (27) can achieve the path constraint defined in Eq. (4), so it can be used for inter- 


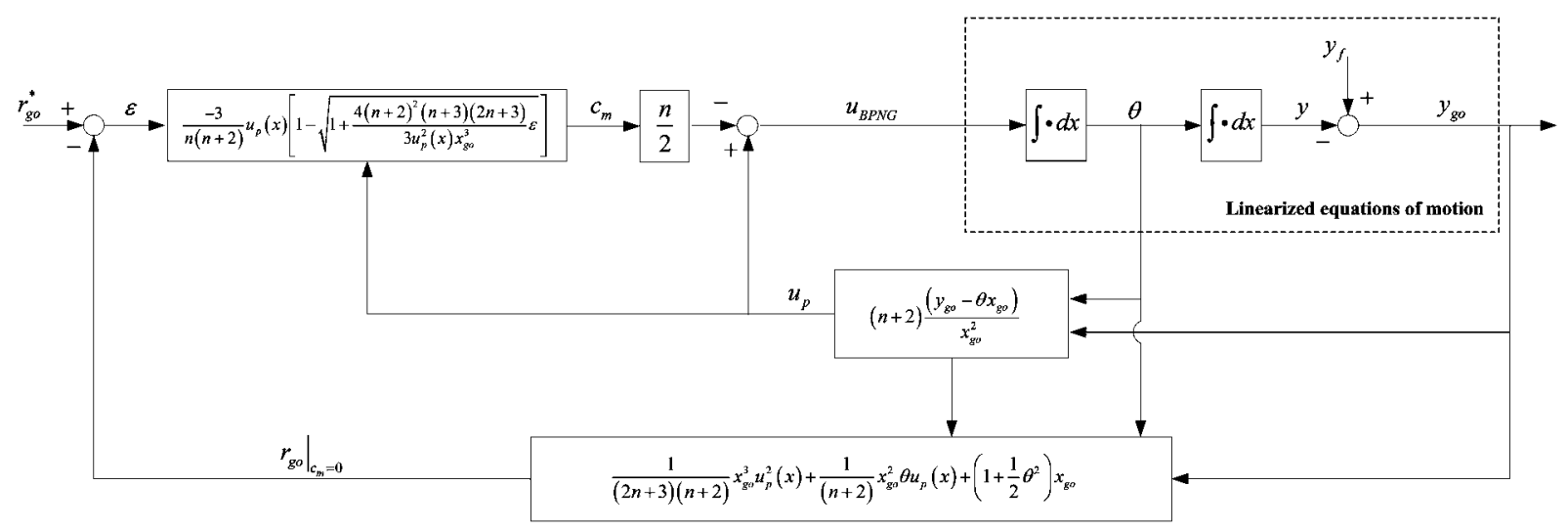

Fig. 2. Guidance loop of impact-time control law.

cepting the target at the desired impact time by employing the $c_{m}$ solution in $u_{\mathrm{BPNG}}$ given by Eq. (13). However, since the $c_{m}$ solution contains the square root term, the $c_{m}$ may have an imaginary solution when $\hat{\varepsilon}<0$. To avoid this unacceptable result, the desired impact time $t_{d}$ should be selected to be larger than $\left.\hat{r}_{\mathrm{go}}\right|_{c_{m}=0} / V$. This requirement implies that the desired distance-to-go, $\hat{r}_{\text {go }}^{*}$, should be larger than the total length of the intercept trajectory generated by $u_{p}(x)$.

\subsection{Guidance command for practical implementation}

From Eq. (27), $c_{m}$ is the constant calculated using the initial homing conditions, but this solution contains an error due to the approximation of $S$. Therefore, in order to reduce the approximation error, the coefficient $c_{m}$ should be applied in a feedback form as follows.

$c_{m}=\frac{-3}{n(n+2)} u_{p}(x)\left[1-\sqrt{1+\frac{4(n+2)^{2}(n+3)(2 n+3)}{3 u_{p}^{2}(x) x_{\mathrm{go}}^{3}} \varepsilon}\right]$

where

$$
\begin{aligned}
\varepsilon= & r_{\mathrm{go}}^{*}-\left.r_{\mathrm{go}}\right|_{c_{m}=0} \\
r_{\mathrm{go}}^{*}= & V\left(t_{d}-t\right) \\
\left.r_{\mathrm{go}}\right|_{c_{m}=0}= & \frac{1}{(2 n+3)(n+2)} x_{\mathrm{go}}^{3} u_{p}^{2}(x) \\
& +\frac{1}{(n+2)} x_{\mathrm{go}}^{2} \theta u_{p}(x)+\left(1+\frac{1}{2} \theta^{2}\right) x_{\mathrm{go}}
\end{aligned}
$$

Note that $c_{m}$ given by Eq. (28) is no longer constant and is updated at each step of $x$.

Using Eqs. (13) and (28), we can finally obtain the guidance command to achieve the terminal zero miss-distance as well as the impact-time control, as shown in Eq. (30).

$$
\begin{aligned}
u_{\mathrm{BPNG}}(x)= & u_{p}(x)-\frac{n}{2} c_{m} \\
= & u_{p}(x)\left[\frac{2 n+7}{2(n+2)}-\frac{3}{2(n+2)}\right. \\
& \left.\times \sqrt{1+\frac{4(n+2)^{2}(n+3)(2 n+3)}{3 u_{p}^{2}(x) x_{\mathrm{go}}^{3}} \varepsilon}\right]
\end{aligned}
$$

From the proposed guidance law in Eq. (30), it is seen that the $u_{\mathrm{BPNG}}$ command is gradually converted to the $u_{p}$ com- mand as the distance-to-go error, $\varepsilon \rightarrow 0$. As discussed in the previous subsection, the desired impact time should be larger than $\left.\hat{r}_{\text {go }}\right|_{c_{m}=0} / V$ to avoid the imaginary solution, so the distance-to-go error at the beginning of the homing phase is always a positive value. In addition, the distanceto-go error gradually decreases and finally reaches the zero as the missile approaches the target. For the practical implementation without numerical instability, therefore, we determine the distance-to-go error by $\varepsilon=\max \left(\varepsilon, \varepsilon_{\min }\right)$, where $\varepsilon_{\min }=-3 u_{p}^{2}(x) x_{\mathrm{go}}^{3} / 4(n+2)^{2}(n+3)(2 n+3)$. Figure 2 illustrates the guidance loop of the proposed impact-time controller given by Eq. (30), which consists of two feedback loops: the inner $u_{p}$ feedback loop is to reduce the missdistance and the outer feedback loop is to reduce the distance-to-go error for achieving the terminal impact time constraint.

To implement the proposed guidance law to a realistic missile system, the guidance command of Eq. (30) should be transformed to the missile acceleration with measurable units in the time domain. Under the assumption that $\theta$ is small and $x_{\mathrm{go}} \gg y_{\mathrm{go}}$, the line-of-sight angle and its derivative can be approximated as

$$
\lambda \approx \frac{y_{\mathrm{go}}}{x_{\mathrm{go}}}, \quad \frac{\mathrm{d} \lambda}{\mathrm{d} x} \approx \frac{\left(y_{\mathrm{go}}-\theta x_{\mathrm{go}}\right)}{x_{\mathrm{go}}^{2}}
$$

Using the definition of $a \triangleq V^{2} u$ and Eq. (31), $a_{p}$ can be expressed as

$a_{p}(t)=V^{2} u_{p}(x)=(n+2) V^{2} \frac{\left(y_{\mathrm{go}}-\theta x_{\mathrm{go}}\right)}{x_{\mathrm{go}}^{2}} \approx(n+2) V \dot{\lambda}$

From Eq. (32), the missile acceleration command corresponding to Eq. (30) in the time domain is derived as

$$
\begin{aligned}
a_{\mathrm{BPNG}}(t)= & V^{2} u_{\mathrm{BPNG}}(x) \\
= & a_{p}(t)\left[\frac{2 n+7}{2(n+2)}-\frac{3}{2(n+2)}\right. \\
& \left.\times \sqrt{1+\frac{4(n+2)^{2}(n+3)(2 n+3)}{3 a_{p}^{2}(t) r^{3}} V^{4} \varepsilon}\right]
\end{aligned}
$$

where $x_{\mathrm{go}} \approx r$ and $r$ is the relative range between the missile and target. The estimated distance-to-go of Eq. (29) can also 
Table 1. Examples of impact-time control law.

\begin{tabular}{|c|c|c|c|}
\hline$n$ & $N$ & Acceleration command, $a_{\mathrm{BPNG}}(t)$ & $\begin{array}{l}\text { Distance-to-go, } \\
\left.\quad r_{\mathrm{go}}\right|_{c_{m}=0}\end{array}$ \\
\hline 0.5 & 2.5 & $2.5 V \dot{\lambda}\left(\frac{8}{5}-\frac{3}{5} \sqrt{1+\frac{350}{3 \cdot(2.5 V \dot{\lambda})^{2} r^{3}} V^{4} \varepsilon}\right.$ & $r\left[1+\frac{1}{8}(\theta-\lambda)^{2}\right]$ \\
\hline 1 & 3 & $3 V \dot{\lambda}\left(\frac{3}{2}-\frac{1}{2} \sqrt{1+\frac{240}{(3 V \dot{\lambda})^{2} r^{3}} V^{4} \varepsilon}\right.$ & $r\left[1+\frac{1}{10}(\theta-\lambda)^{2}\right.$ \\
\hline 2 & 4 & $4 V \dot{\lambda}\left(\frac{11}{8}-\frac{3}{8} \sqrt{1+\frac{2240}{3 \cdot(4 V \dot{\lambda})^{2} r^{3}}} V^{4} \varepsilon\right.$ & $r\left[1+\frac{1}{14}(\theta-\lambda)^{2}\right.$ \\
\hline
\end{tabular}

be expressed in terms of the line-of-sight angle and the relative range as

$$
\begin{aligned}
\left.r_{\mathrm{go}}\right|_{c_{m}=0} & =x_{\mathrm{go}}\left[1+\frac{1}{2(2 n+3)}(\theta-\lambda)^{2}+\frac{1}{2} \lambda^{2}\right] \\
& \approx r\left[1+\frac{1}{2(2 n+3)}(\theta-\lambda)^{2}\right]
\end{aligned}
$$

Note that the $a_{p}$ command corresponding to $u_{p}$ is the PNG law with navigation constant of $n+2$; that is, $a_{p}=N V \dot{\lambda}$ because $n+2=N$ in Eq. (7). Therefore, the proposed law given by Eq. (33) is converged to the PNG as $\varepsilon \rightarrow 0$ and $r \rightarrow 0$. Examples of the proposed impact-time control law for several values of $n$ are shown in Table 1. It is noted that the command with $n=1(N=3)$ is identical to the suboptimal guidance law proposed in Ref. 1).

\section{Numerical Examples}

To investigate the characteristics and performance of the proposed impact-time control law, numerical simulations with the nonlinear equations of motion in Eq. (1) and the acceleration command of Eq. (33) are performed. In these simulations, we assume that the missile has a constant speed of $300 \mathrm{~m} / \mathrm{s}$, and that the initial fight path angle and the desired impact time are $30^{\circ}$ and $45 \mathrm{~s}$, respectively. The initial missile and stationary target positions in the inertial reference frame $(X-Y)$ are $(0,0) \mathrm{m}$ and $(10,000,0) \mathrm{m}$, respectively. These engagement conditions are summarized below in Table 2, and all simulations are terminated when the relative range, $r$, is less than $0.5 \mathrm{~m}$.

Using these simulation conditions without the terminal impact time constraint and the conventional PNG law with different navigation constants $N=2.5,3.0,4.0,5.0$, we first perform the simulations. The results are presented in Figs. 3 and 4. As shown in Fig. 3, all the missiles driven by the PNG laws impact the target at roughly $34 \mathrm{~s}$, even though the PNG with larger navigation constant generates the shorter homing trajectory. Figure 4 presents the acceleration commands of the PNG laws. We can see from this figure that the larger navigation constant produces the higher command at the beginning of the flight and the lower command near the end of the flight. We can also see that the missile's maximum acceleration required to hit the target increases as the navigation constant is increased from 2.5 to 5.0. Note that the total flight time of the PNG laws, which
Table 2. Simulation conditions.

\begin{tabular}{ll}
\hline \multicolumn{1}{c}{ Parameters } & \multicolumn{1}{c}{ Values } \\
\hline Target position, $\left(x_{f}, y_{f}\right)$ & $(10,000,0) \mathrm{m}$ \\
Initial missile position, $\left(x_{0}, y_{0}\right)$ & $(0,0) \mathrm{m}$ \\
Missile velocity, $V$ & $300 \mathrm{~m} / \mathrm{s}$ \\
Initial flight path angle, $\theta_{0}$ & $30 \mathrm{deg}$ \\
Desired impact time, $t_{d}$ & $45 \mathrm{~s}$ \\
Guidance gain, $n$ & $n=0.5,1.0,2.0,3.0$ \\
\hline
\end{tabular}
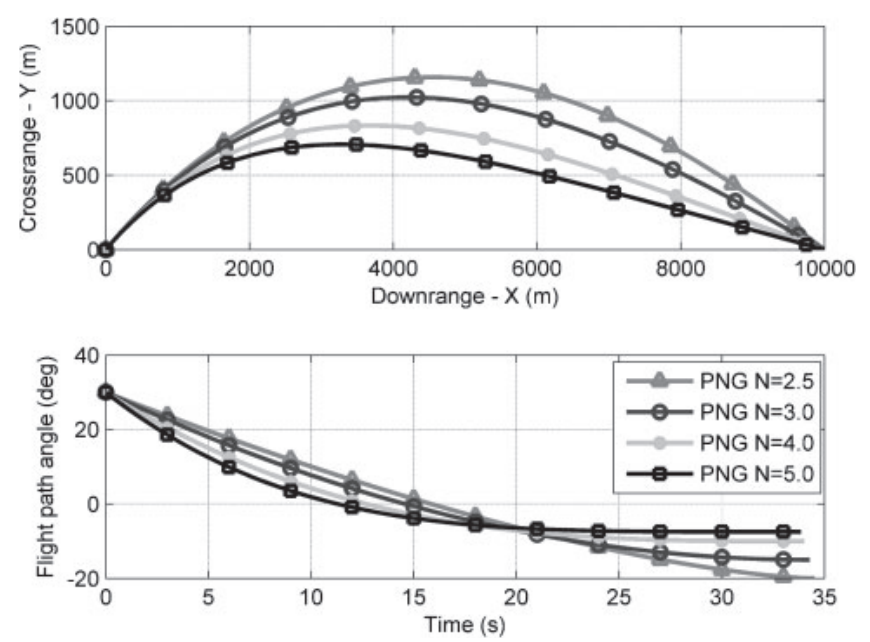

Fig. 3. Trajectories and flight path angles using PNG.

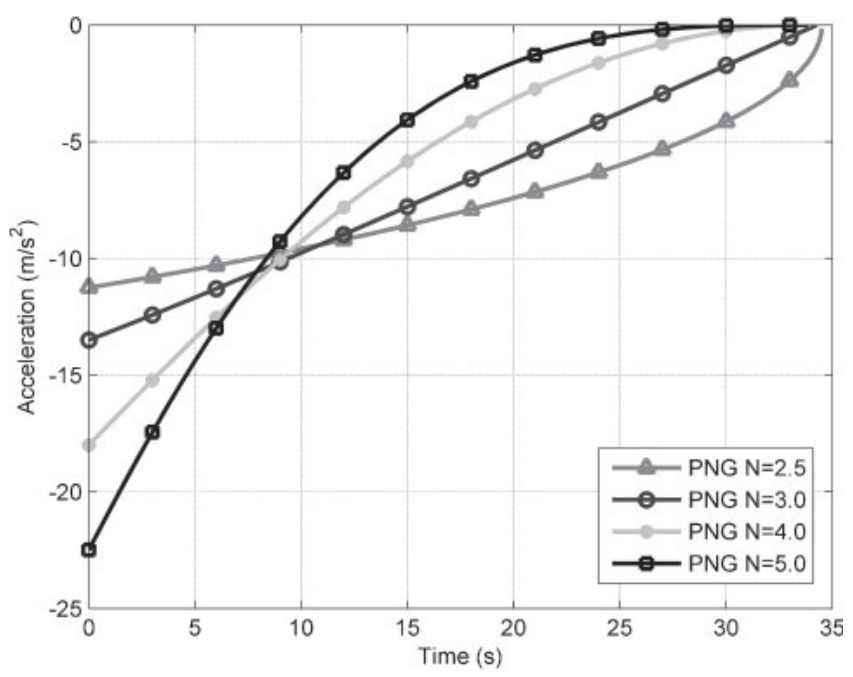

Fig. 4. Acceleration commands of PNG.

can be estimated by $\left.\hat{r}_{\mathrm{go}}\right|_{c_{m}=0} / V$, is quite different from the desired impact time, so the PNG cannot achieve the terminal impact time constraint and the flight time of the PNG is dependent on the initial engagement conditions.

Next, we perform the numerical simulations in which the terminal impact time constraint, $t_{d}=45 \mathrm{~s}$, is imposed. The proposed impact-time control law of Eq. (33) and estimated distance-to-go of Eq. (34) are used for these simulations, and the different guidance gains $n$ are selected as in Table 2 .

Figures 5 to 7 present the simulation results: the intercept trajectories, flight path angles, acceleration commands and distance-to-go errors resulting from the proposed impact- 

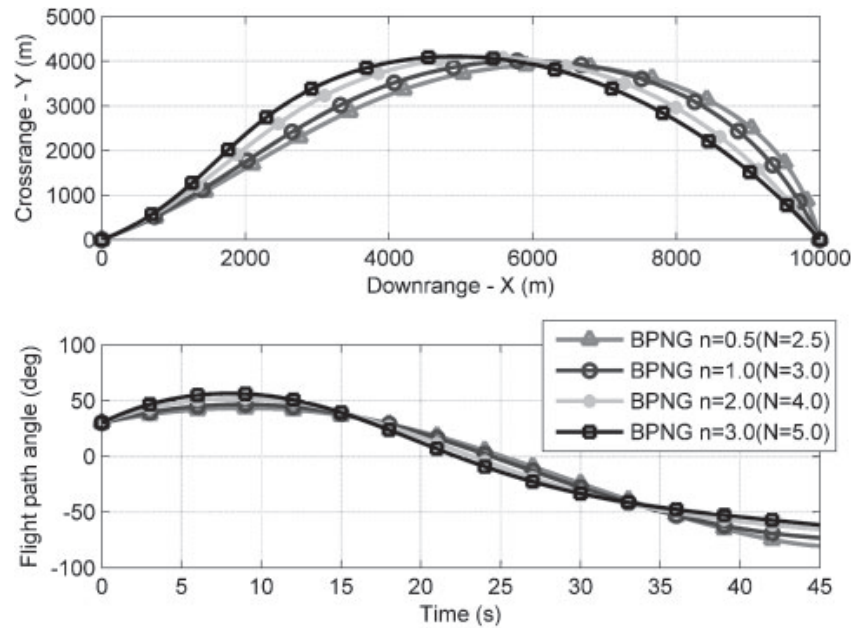

Fig. 5. Trajectories and flight path angles using BPNG.
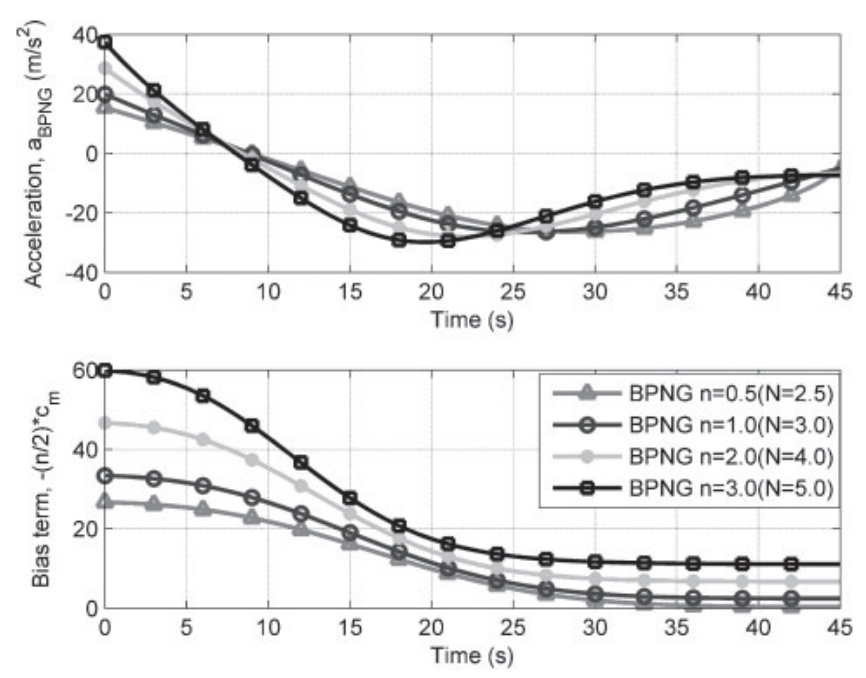

Fig. 6. Acceleration commands and bias terms of BPNG.

time control law with various $n$ gains. As shown in the figures, for all the cases, the proposed BPNG law enables the missile to hit the target at the designated impact time, $45 \mathrm{~s}$, by increasing the length of the intercept trajectory. The bias terms, defined as $-(n / 2) c_{m}$ in the proposed law, slowly decrease because the distance-to-go errors are reduced to zero as the missile approaches the target. However, since some small value of the distance-to-go errors remains during the terminal phase of the flight except at the instant of interception, the bias terms do not converge to zero exactly. From the figures, it can be seen that increasing the guidance gain $n$ increases the initial missile acceleration command, whereas it decreases the acceleration command in the terminal homing phase. It can also be seen that increasing the guidance gain increases both the maximum acceleration and the bias term for trajectory shaping, and it makes the distance-to-go error converge to zero more quickly. As compared with the PNG, the proposed BPNG to control the impact time requires more acceleration capability.

As mentioned in the previous section, the proposed impact-time control law with $n=1.0(N=3.0)$ is the same form as the suboptimal impact-time controller suggested in

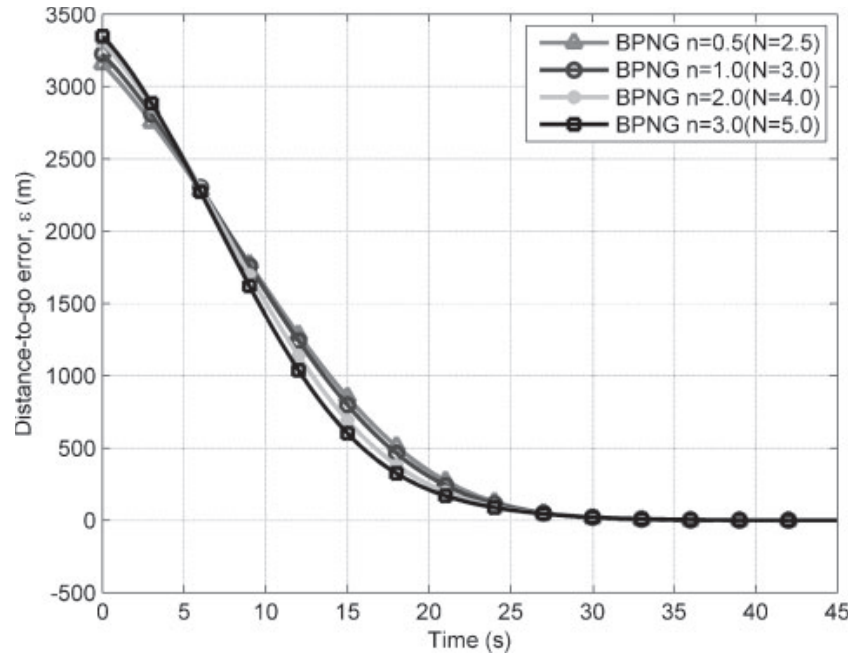

Fig. 7. Distance-to-go errors of BPNG.

Table 3. Comparisons of costs.

\begin{tabular}{ccccc}
\hline$n(N)$ & $0.5(2.5)$ & $1.0(3.0)$ & $2.0(4.0)$ & $3.0(5.0)$ \\
\hline$J=\int a^{2}(t) \mathrm{d} t$ & $1.441 \times 10^{4}$ & $1.404 \times 10^{4}$ & $1.478 \times 10^{4}$ & $1.623 \times 10^{4}$ \\
\hline
\end{tabular}

Ref. 1), which minimizes the energy cost function subject to the terminal impact time constraint. To verify this result, we calculate the cost function defined as the integral of the square of the acceleration command. Table 3 shows the energy costs for each guidance gain $n$ with $t_{d}=45 \mathrm{~s}$ constraint. As can be seen in Table 3, the proposed BPNG with $n=1.0(N=3.0)$ has the lowest cost. It can also be seen that the cost increases as the gain $n$ increases. Although the proposed law with $n=0.5(N=2.5)$ requires smaller acceleration capability than other cases, the law with $n=0.5(N=2.5)$ has higher cost than the case of $n=1.0$ $(N=3.0)$. Therefore, we can say that the BPNG with $n=1.0(N=3.0)$ is the suboptimal impact-time control law which minimizes the energy cost and achieves the terminal impact time constraint.

We also perform the numerical simulations with different impact times, $t_{d}=40,50,60 \mathrm{~s}$, and different gains $n=0.5$, 1.0, 2.0, 3.0. The other parameters are the same as in Table 2. Missile trajectories, flight path angles, acceleration commands of the proposed impact-time control law for all the cases are presented in Figs. 8 to 15 . As shown in these figures, the results prove the capability of the proposed law for satisfying the various impact time constraints, even though the proposed law is derived on the basis of the linearized equations and some assumptions.

Since the real missile systems have a maximum acceleration limit, large miss-distances or large impact-time errors may be caused by the acceleration saturation. Therefore, simulations with the acceleration limitation $|a| \leq 30 \mathrm{~m} / \mathrm{s}^{2}$ and previous conditions are performed for various impact times and guidance gains. Figure 16 shows the simulation results of the impact-time error, where the error is the difference between the desired impact time and the final intercept 

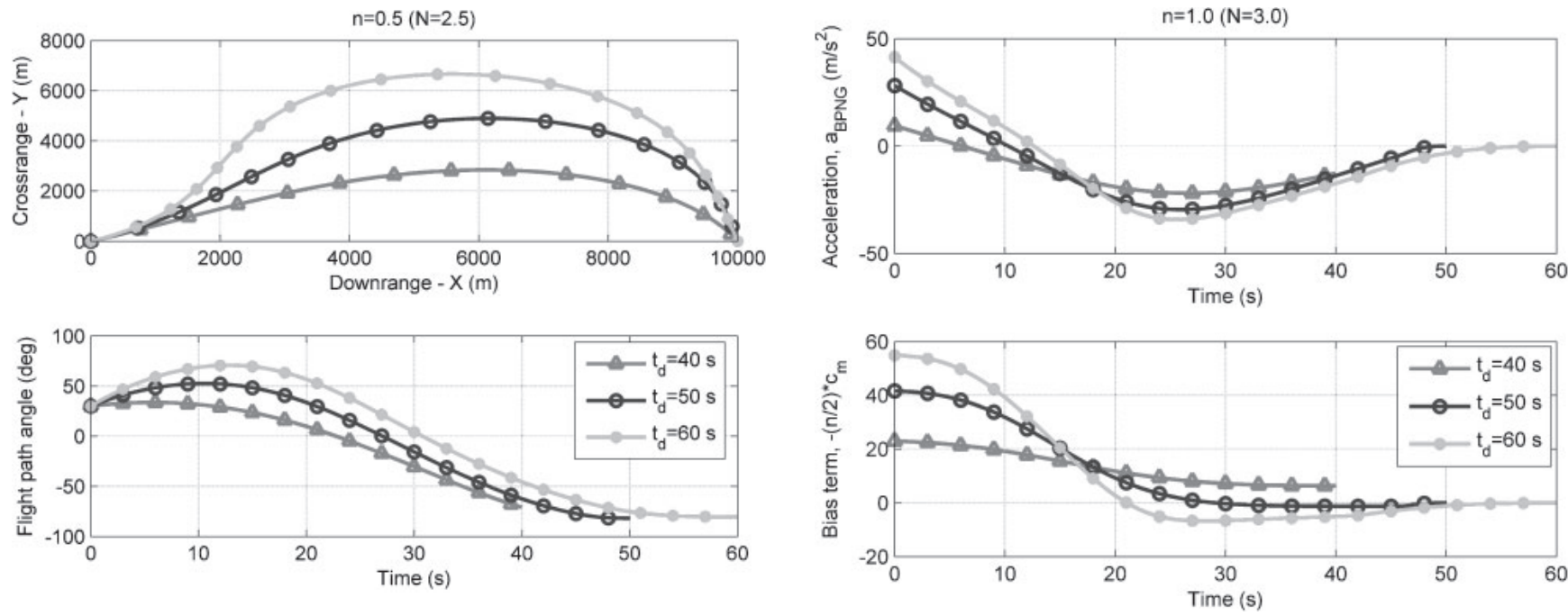

Fig. 8. Trajectories and flight path angles of BPNG with $n=0.5$ for various desired impact times.

Fig. 11. Acceleration commands and bias terms of BPNG with $n=1.0$ for various desired impact times.
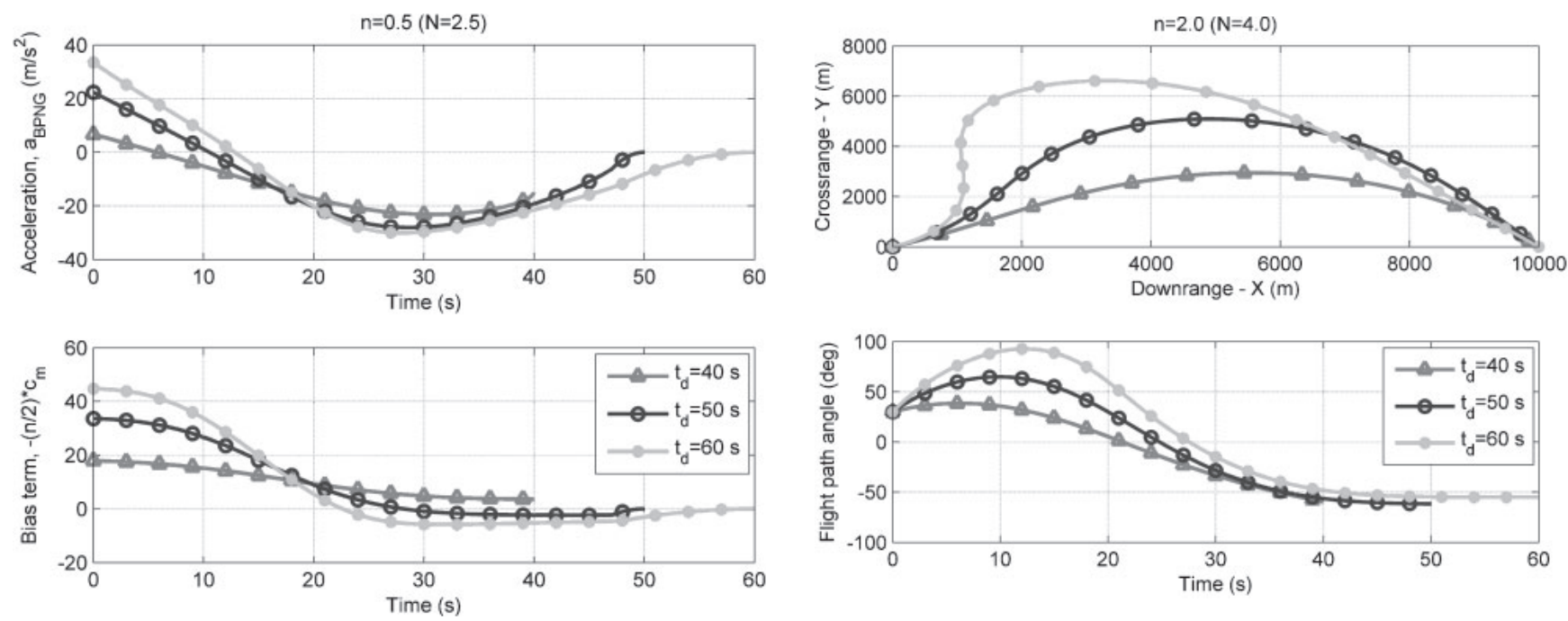

Fig. 9. Acceleration commands and bias terms of BPNG with $n=0.5$ for various desired impact times.

Fig. 12. Trajectories and flight path angles of BPNG with $n=2.0$ for various desired impact times.
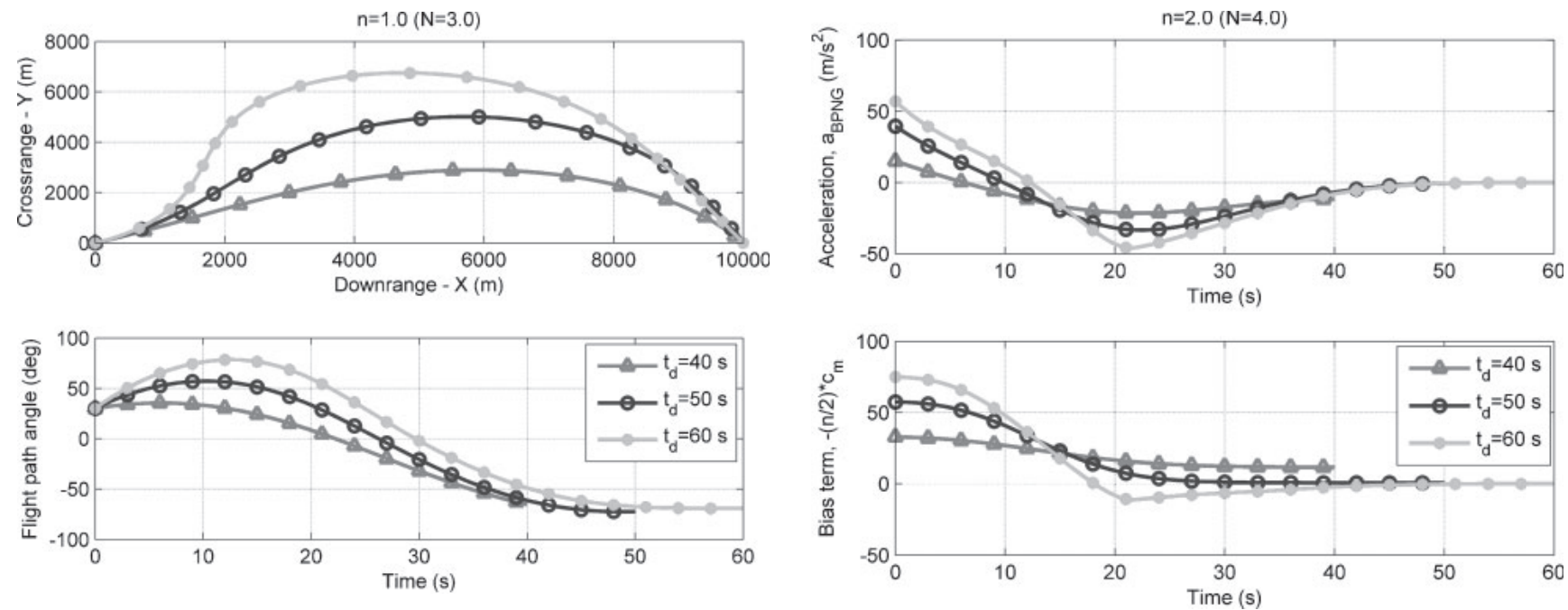

Fig. 10. Trajectories and flight path angles of BPNG with $n=1.0$ for various desired impact times.

Fig. 13. Acceleration commands and bias terms of BPNG with $n=2.0$ for various desired impact times. 

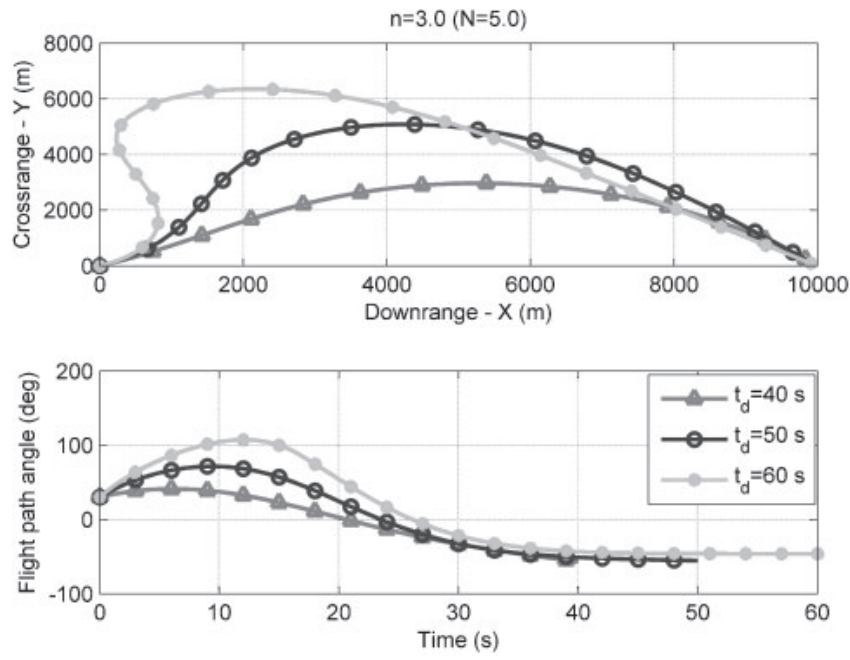

Fig. 14. Trajectories and flight path angles of BPNG with $n=3.0$ for various desired impact times.
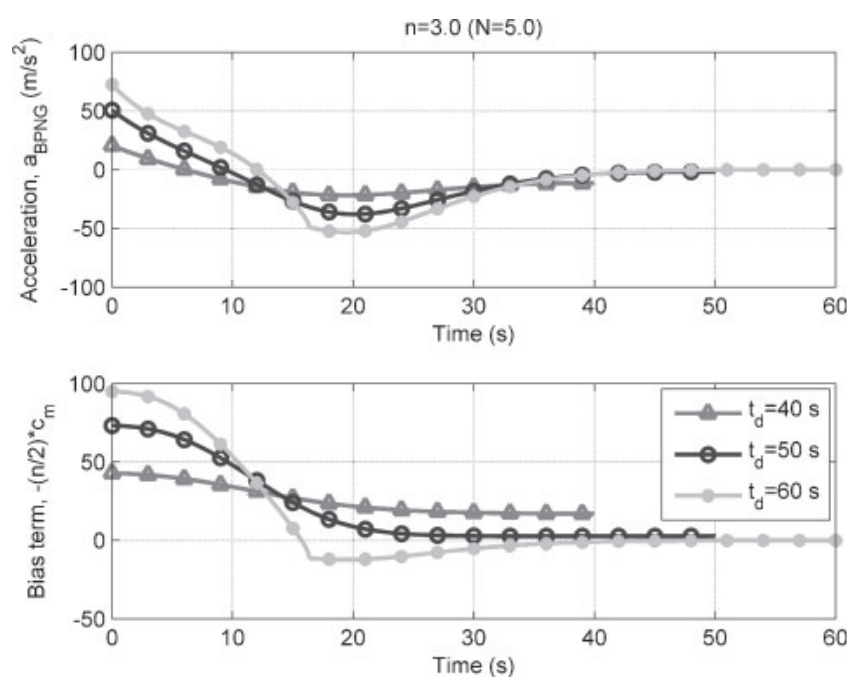

Fig. 15. Acceleration commands and bias terms of BPNG with $n=3.0$ for various desired impact times.

time at which $r<0.5 \mathrm{~m}$. From Fig. 16, it is observed that the impact-time error increases as either the guidance gain or the desired impact time increases because the missile acceleration saturates. Therefore, in order to avoid acceleration saturation and reduce the impact time error, proper guidance gain should be used in the proposed impact-time control law or the missile should have sufficient acceleration capability.

\section{Conclusion}

In this paper, a BPNG law was proposed for intercepting stationary targets at desired impact time. To derive the feedback guidance law for the impact-time control, we introduced a polynomial function of the downrange-to-go with two coefficients which were determined to satisfy the terminal constraints. The final form of the proposed guidance law was represented as a combination of the well-known PNG law with arbitrary navigation constant

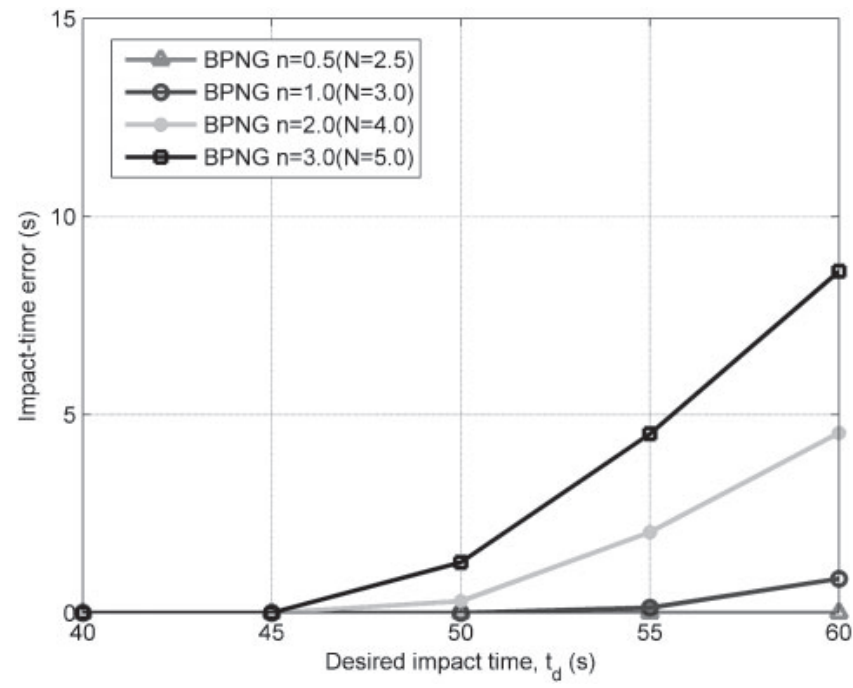

Fig. 16. Impact-time errors due to acceleration saturation.

$N>2$ and the bias term to achieve the desired impact time constraint. In order to reduce the distance-to-go error and approximation error, the bias term was implemented in a feedback form, so it was updated at each time step. Since the proposed impact-time control law generates different intercept trajectories and different acceleration command profiles according to the choice of the navigation constant, a designer can choose the proper guidance law in consideration of the missile system capabilities and operating conditions. The guidance performance and characteristics of the proposed law were demonstrated through numerical simulations with various engagement conditions. The simulation results indicated that the proposed impact-time control law can achieve the zero miss-distance as well as terminal impact time constraint with negligible errors. Even if the maximum acceleration capability of the missile is limited, the proposed guidance law can provide satisfactory performance by choosing the proper navigation constant. In general, the missile has several issues such as aerodynamic effects, autopilot lag and sensor noises in addition to acceleration capability limitations. Considering these issues, more detailed guidance performance analyses of the proposed impact-time control law should be performed in future studies.

\section{References}

1) Jeon, I. S., Lee, J. I. and Tahk, M. J.: Impact-Time-Control Guidance Law for Anti-Ship Missiles, IEEE Trans. Control Syst. Technol., 14, 2 (2006), pp. 260-266.

2) Lee, J. I., Jeon, I. S. and Tahk, M. J.: Guidance Law to Control Impact Time and Angle, IEEE Trans. Aerospace Electron. Syst., 43, 1 (2007), pp. 301-310.

3) Alighanbari, M., Kuwata, Y. and How, J. P.: Coordination and Control of Multiple UAVs with Timing Constraints and Loitering, 2003 American Control Conference, Denver, CO, 2003.

4) Beard, R. W., McLain, T. W. and Goodrich, M.: Coordinated Target Assignment and Intercept for Unmanned Air Vehicles, 2002 IEEE International Conference on Robotics \& Automation, Washington, DC, 2002.

5) Curtis, J. W. and Murphey, R.: Simultaneous Area Search and Task 
Assignment for a Team of Cooperative Agents, AIAA Guidance, Navigation, and Control Conference, Austin, TX, 2003.

6) McLain, T. W., Chandler, P. R., Rasmussen, S. and Pachter, M.: Cooperative Control of UAV Rendezvous, 2001 American Control Conference, Arlington, VA, 2001.

7) Ryoo, C. K., Kim, Y. H. and Tahk, M. J.: Optimal UAV Formation Guidance Laws with Timing Constraint, Int. J. Syst. Sci., 37, 6 (2006), pp. 415-427.

8) Richards, A. and How, J. P.: Model Predictive Control of Vehicle Maneuvers with Guaranteed Completion Time and Robust Feasibility, 2003 American Control Conference, Denver, CO, 2003.

9) Jeon, I. S., Lee, J. I. and Tahk, M. J.: Homing Guidance Law for Cooperative Attack of Multiple Missiles, J. Guid. Control Dynam., 33, 1 (2010), pp. 275-280.

10) Lee, J. I., Jeon, I. S. and Tahk, M. J.: Guidance Law Using Augmented Trajectory-Reshaping Command for Salvo Attack of Multiple Missiles, UKACC International Control Conference 2006, Glasgow, Scotland, 2006.

11) Zhang, Y., Yu, D., Zhang, Y. A. and Wu, Y.: An Impact-Time-Control Guidance Law for Multi-Missiles, Intelligent Computing and Intelligent Systems, ICIS 2009 Conference, Shanghai, 2009.

12) Jung, B. and Kim, Y.: Guidance Laws for Anti-Ship Missiles Using
Impact Angle and Impact Time, AIAA Guidance, Navigation, and Control Conference, Keystone, Colorado, 2006.

13) Harl, N. and Balakrishnan, S. N.: Impact Time and Angle Guidance with Sliding Mode Control, AIAA Guidance, Navigation, and Control Conference, Chicago, Illinois, 2009.

14) Kreindler, E.: Optimality of Proportional Navigation, AIAA J., 11, 6 (1973), pp. 878-880.

15) Guelman, M.: The Closed-Form Solution of True Proportional Navigation, IEEE Trans. Aerospace Electron. Syst., 12, 4 (1976), pp. 472-482.

16) Becker, K.: Closed-Form Solution of Pure Proportional Navigation, IEEE Trans. Aerospace Electron. Syst., 26, 3 (1990), pp. 526-533.

17) Bryson Jr., A. E. and Ho, Y.-C.: Applied Optimal Control, Wiley, New York, 1975.

18) Lewis, F. L.: Optimal Control, Wiley, New York, 1986.

19) Kreyszig, E.: Advanced Engineering Mathematics, 9th Ed., Wiley, Hoboken, 2005.

20) Zarchan, P.: Tactical and Strategic Missile Guidance, 3rd Ed., AIAA, Washington, DC, 1997.

21) Lin, C. F.: Modern Navigation, Guidance, and Control Proceeding, Prentice Hall, New Jersey,1991. 